\title{
An Ultra-Wideband Millimeter-Wave Phased Array
}

\author{
Markus H. Novak*, Félix A. Miranda ${ }^{\dagger}$, John L. Volakis* \\ *ElectroScience Laboratory, The Ohio State University, Columbus, OH, USA \\ ${ }^{\dagger}$ NASA Glenn Research Center, Cleveland, OH, USA
}

\begin{abstract}
Wideband millimeter-wave arrays are of increasing importance due to their growing use in high data rate systems, including $5 \mathrm{G}$ communication networks. In this paper, we present a new class of ultra-wideband millimeter wave arrays that operate from nearly $20 \mathrm{GHz}$ to $90 \mathrm{GHz}$. The array is based on tightly coupled dipoles. Feeding designs and fabrication challenges are presented, and a method for suppressing feed resonances is provided.
\end{abstract}

\section{INTRODUCTION}

There is growing interest for a multitude of communication capabilities to be integrated into smaller sized platforms. Small satellites, unmanned aerial vehicles, cars, body-warn sensor systems and handsets are example applications. These small platforms typically do not have sufficient physical space to house a large number of antenna systems, implying a need for a spectrally agile, multi-function aperture. This can be accomplished using electronically scanned, ultra-wideband arrays. One such array is the Tightly Coupled Dipole Array (TCDA). TCDAs have been previously demonstrated to achieve up to 8:1 bandwidth and $>60^{\circ}$ scanning [1]-[3].

So far, most TCDA designs have focused on operations below $10 \mathrm{GHz}$. However, the fifth generation mobile architecture $(5 \mathrm{G})$ leans on access to the millimeter-wave spectrum for high capacity communication links. To achieve access to these frequencies, we demonstrate a novel TCDA feed and element design, optimized for simple fabrication at millimeter-wave frequencies. Operating from $24 \mathrm{GHz}$ to $86 \mathrm{GHz}$, this array provides access to candidate $5 \mathrm{G}$ frequencies at $26.4 \mathrm{GHz}$ and $58.6 \mathrm{GHz}$. Operation at the ISM bands centered at $24.125 \mathrm{GHz}$ and $61.25 \mathrm{GHz}$ is also included. We will show that the array can be easily extended to dual polarizations, and is capable of wide angle scanning in all planes. The full array can be fabricated in a single step, using very low cost techniques, on low loss laminates.

\section{DESIGN CONCEPT}

Generally, TCDAs achieve very wide bandwidths by implementing a higher order matching network in the form of a balun, integrated within the volume of the array. But at millimeter-wave frequencies, the complexity of the feeding circuitry conflicts with the inherently low-profile nature of the array topology. The latter can be as little as 1/16 of a wavelength at the lowest frequency of operation, implying a limitation based on the achievable feature size of the

This work was supported by a NASA Space Technology Research Fellowship, under grant \#NNX13AL48H.

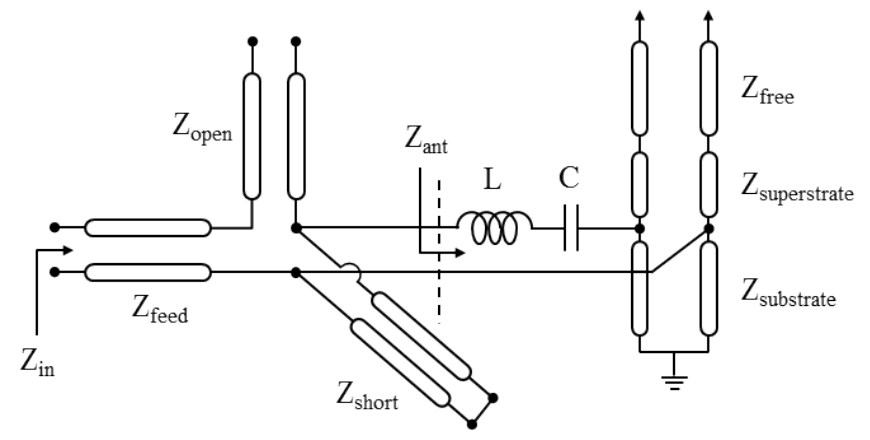

Fig. 1. Transmission line model of a Tightly Couple Dipole Array and feeding network. The reactance $L$ is caused by the dipole's inherent inductance, and $C$ is due to the resultant capacitive coupling between neighboring elements in the array.

selected fabrication technique. As such, the arrays [1] and [2] are limited to operation below $5 \mathrm{GHz}$. Recently, in [3], a simplified TCDA was designed and fabricated to operate up to $18.5 \mathrm{GHz}$. We note the array [3] can be linearly scaled up to $49 \mathrm{GHz}$ while remaining within commercial Printed Circuit Board $(\mathrm{PCB})$ fabrication capabilities $(\approx 75 \mu \mathrm{m}$ feature size).

The TCDA array can be represented by the equivalent circuit model shown in Fig. 1. In this model, the balun comprises a series open circuit and shunt short circuit, and the element is represented as a series dipole inductance and interelement capacitance. For optimal bandwidth, the characteristic impedance $Z_{\text {open }}$ should be very small (viz., $<20 \Omega$ ), while the impedances $Z_{\text {feed }}$ and $Z_{\text {short }}$ are high (viz., $>100 \Omega$ ). This ratio of impedances is increasingly difficult to achieve as the minimum realizable feature size becomes a significant fraction of the wavelength. As such, it is necessary to simplify the feeding network so that it is realizable in the millimeterwave regime. From [4], it is known that the virtual short formed from the very low impedance open circuit can be replaced with a direct short. This does not significantly impact the transformers bandwidth, as long as the input and output impedances are still well matched. However, as this condition cannot be guaranteed in the aperture environment, we expect a reduction in the maximum achievable bandwidth of the array [5].

Using only vias and two metal layers above the groundplane, this circuit model can be implemented in a periodic unit cell, as shown in Fig. 2. The element is fed from pin A. Recalling that signals do not propagate on the surface of the 

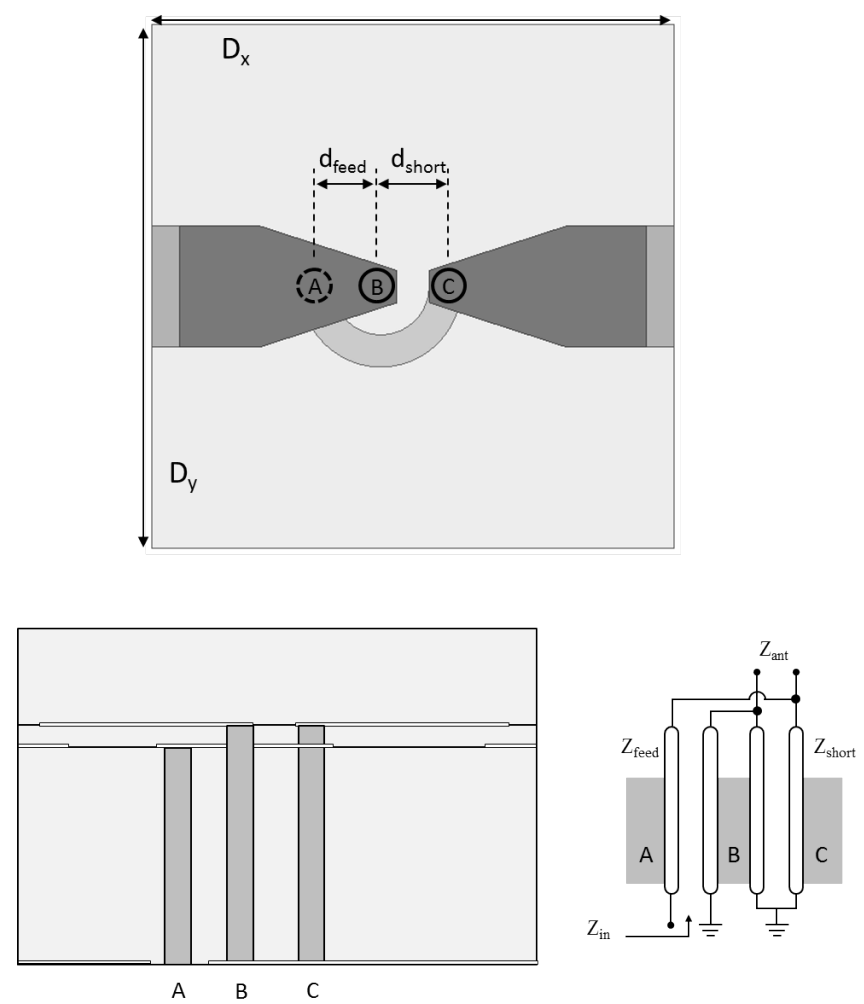

Fig. 2. Top-down view of the periodic unit cell (top). Dark grey shapes exist on the top metal layer, while light grey shapes exist on the lower metal layer. Side view showing the relative depth of vias A, B, and C (bottom left); note that vias $\mathrm{B}$ and $\mathrm{C}$ short the dipoles to the groundplane, while the feed via, $\mathrm{A}$, only extends to the lower metal layer. An equivalent transmission line model is shown at the bottom right.

conductor, but rather in the space between them, we observe that this structure forms two transmission lines opposite to the center conductor. The associated equivalent circuit model is shown at the bottom right of Fig. 2, where $Z_{a n t}$ is unchanged from Fig. 1 . The impedance $Z_{\text {feed }}$ is then primarily determined by the separation $d_{\text {feed }}$, and likewise $Z_{\text {short }}$ is determined by $d_{\text {short }}$.

\section{PREDicting SpuRious RESONANCES}

VSWR plots from a full wave simulation of the model in Fig. 2 are shown in Fig. 3. These were generated by a selecting a unit cell size $\left(D_{x}\right.$ and $\left.D_{y}\right)$ of $1.65 \mathrm{~mm}$ and groundplane depth of $1 \mathrm{~mm}$ (substrate $\epsilon_{r}=2.1$ ). We note that the array exhibits wideband behavior, but with a spurious resonance at $48 \mathrm{GHz}$ (central peak in Fig. 3). This is a result of an imbalance in the magnitude of the currents flowing on the dipole pins, B and C. Based on the geometry of the array, this net vertical current can excite strong fields perpendicular to the groundplane, in the substrate. We also note that the field distribution along the diagonal between neighboring cells resembles a waveguide $\mathrm{TE}_{10}$ mode, reaching a maximum between elements and tapering to zero at the grounded conductors. As such, we can estimate the resonant frequency of a given geometry using the well-known relation

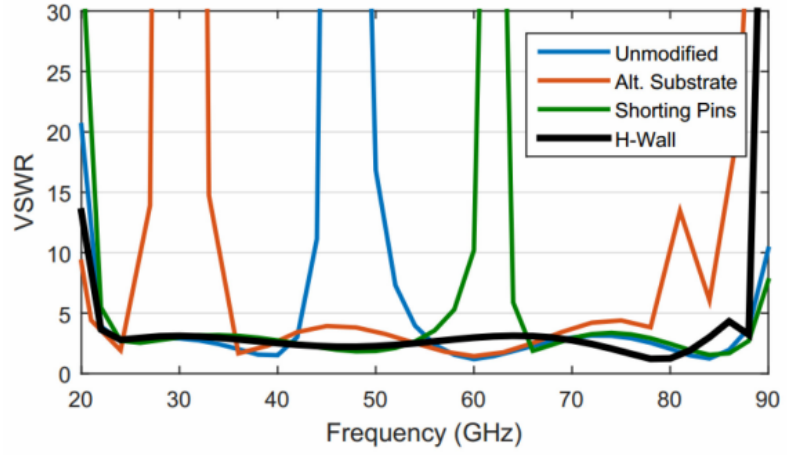

Fig. 3. VSWR response from full-wave simulation of the infinite millimeterwave array, showing the resonances of the 1) unmodified array (center, blue), 2) a high dielectric constant substrate (left, red), 3) the inclusion of shorting pins (right, green), and 4) inclusion of a conducting H-wall (black).

$$
f_{r}=\frac{c}{2 D \sqrt{\epsilon_{r}}}
$$

Here $f_{r}$ is the frequency of resonance, $\mathrm{D}$ is the distance between the grounded conductors, and $\epsilon_{r}$ is the relative dielectric constant of the substrate. Substituting the values $D=\sqrt{\left(D_{x}-d_{\text {feed }}\right)^{2}+D_{y}^{2}}=2.2 \mathrm{~mm}$ and $\epsilon_{r}=2.1$, we calculate $f_{r}$ as $46 \mathrm{GHz}$. This value agrees closely with the peak observed in Fig. 3. Clearly, to eliminate this resonance, we must force $f_{r}$ to occur either above or below the band of interest.

While the onset of $f_{r}$ is easily pushed out of band by adjusting the value of $D_{x}$ and $D_{y}$ (the unit cell spacing) this is not desirable, as it causes oversampling of the array when decreased and allows grating lobes when increased. Instead, we may try to lower $f_{r}$ by using a substrate of very high $\epsilon_{r}$. Substituting $\epsilon_{r}=6$ into 1 , we see $f_{r}$ is indeed lowered to $28 \mathrm{GHz}$, corresponding to the leftmost peak in Fig. 3. Likewise, we can use 1 to calculate a sufficiently high $\epsilon_{r}$ to eliminate the resonance completely (i.e. set $f_{r}=18 \mathrm{GHz}$ ), yielding $\epsilon_{r}>16$. While the calculated value is theoretically achievable, it is not practical and would cause detrimental surface waves when the array is scanned.

Instead of using a high $\epsilon_{r}$ for the substrate, we will increase the resonant frequency by adding shorting pins from the dipole edges to the groundplane. This approach will now allow the same mode to form diagonally, between the shorting pin and the grounded conductor of a neighboring element. This diagonal path would have an approximate length $1.78 \mathrm{~mm}$, resulting in $f_{r}=58 \mathrm{GHz}$. This agrees closely with the rightmost peak in Fig. 3 .

The occurrence of this resonance can be pushed higher, and ultimately out of the band, by replacing the single shorting pin by a continuous conducting wall, perpendicular to the primary dipole current (solid curve, Fig. 3). This is called an H-wall, and can be implemented either with a series of shorting pins, which must be mutually connected to avoid monopole radiation, or by including an orthogonal polarization 


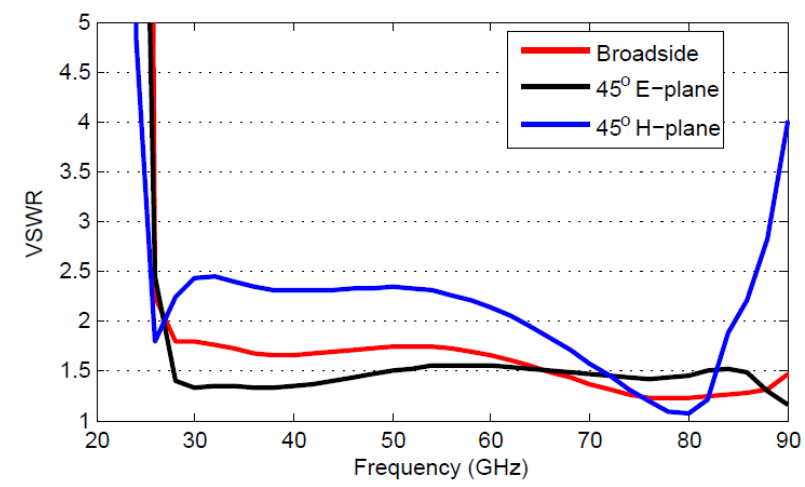

Fig. 4. VSWR response from full-wave simulation of the optimized infinite millimeter-wave array, demonstrating a broadside (red) VSWR $<1.8$ across 26-90 GHz. Likewise, scanning to $\pm 45^{\circ}$ in the E- (black) and $\mathrm{H}$ - (blue) planes shows a well-matched response across the band.

to accomplish the same purpose. The dual polarized unit cell would support a resonant mode between orthogonal feeds with a theoretical $D=0.95 \mathrm{~mm}$, corresponding to a resonant frequency of $109 \mathrm{GHz}$, well outside the operating band.

\section{ImPEDANCE MATChing AND FABRicAtion}

We can conclude from the VSWR curves in Fig. 3 that the array now operates resonance-free across the band. However, there is significant mismatch in this non-optimized model. Specifically, the VSWR can reach just below 5 at some frequencies. Therefore, it is important to further improve wideband matching. Wideband impedance matching to a $50 \Omega$ input can be accomplished through an additional feeding network elements integrated above groundplane, as is demonstrated in Fig. 4. The details of this feeding network, and associated material selection as well as fabrication considerations, will be discussed in the presentation.

\section{REFERENCES}

[1] J. Doane, K. Sertel, and J. L. Volakis, "A wideband, wide scanning tightly coupled dipole array with integrated balun (TCDA-IB)," IEEE Trans. Ant. and Propag., vol. 61, no. 9, pp. 4538-4548, 2013.

[2] W. F. Moulder, K. Sertel, and J. L. Volakis, "Superstrate-enhanced ultrawideband tightly coupled array with resistive FSS," IEEE Trans. Ant. and Propag., vol. 60, no. 9, pp. 4166-4172, 2012.

[3] M. H. Novak, F. A. Miranda, and J. L. Volakis, "Ultra-wideband phased array antennas for satellite communications up to $\mathrm{Ku}$ - and Ka-band," IEEE Trans. Ant. and Propag., In review.

[4] F. C. de Ronde, "A new class of microstrip directional couplers," in $G$ MTT 1970 Int. Microwave Symp., 1970.

[5] J. P. Doane, K. Sertel, and J. L. Volakis, "Optimum design for a broadband microstrip balun,” Electronics Letters, vol. 48, no. 10, p. 540, 2012. 